\title{
A Portfolio Solver for Answer Set Programming: Preliminary Report
}

\author{
M. Gebser, R. Kaminski, B. Kaufmann, T. Schaub, M. Schneider, and S. Ziller \\ Institut für Informatik, Universität Potsdam
}

\begin{abstract}
We propose a portfolio-based solving approach to Answer Set Programming (ASP). Our approach is homogeneous in considering several configurations of the ASP solver clasp. The selection among the configurations is realized via Support Vector Regression. The resulting portfolio-based solver claspfolio regularly outperforms clasp's default configuration as well as manual tuning.
\end{abstract}

\section{Introduction}

Answer Set Programming (ASP; [1]) has become a prime paradigm for declarative problem solving due to its combination of an easy yet expressive modeling language with high-performance Boolean constraint solving technology. In fact, modern ASP solvers like clasp [2] match the performance of state-of-art satisfiability (SAT) checkers, as demonstrated during the last SAT competition in 2009. Unfortunately, there is a price to pay: despite its theoretical power [3], modern Boolean constraint solving is highly sensitive to parameter configuration. In fact, we are unaware of any true application on which clasp is run in its default settings. Rather, in applications, "black magic" is used to find suitable search parameters. Although this is well-known and also exploited in the SAT community, it is hardly acceptable in an ASP setting for the sake of declarativity. The most prominent approach addressing this problem in SAT is satzilla [4], aiming at selecting the most appropriate solver for a problem at hand.

Inspired by satzilla, we address the lack of declarativity in ASP solving by exploring a portfolio-based approach. To this end, we concentrate on the solver clasp and map a collection of instance features onto an element of a portfolio of distinct clasp configurations. This mapping is realized by appeal to Support Vector Regression [5]. In what follows, we describe the approach and architecture of the resulting claspfolio system. We further provide an empirical analysis contrasting claspfolio's performance with that of clasp's default setting as well as the manually tuned settings used during the 2009 ASP competition. In addition, we compare the approach of claspfolio with paramils [6], a tool for parameter optimization based on local search.

\section{Architecture}

Given a logic program, the goal of claspfolio is to automatically select a suitable configuration of the ASP solver clasp. In view of the huge configuration space, the attention is

\footnotetext{
* Affiliated with Simon Fraser University, Canada, and Griffith University, Australia.
} 


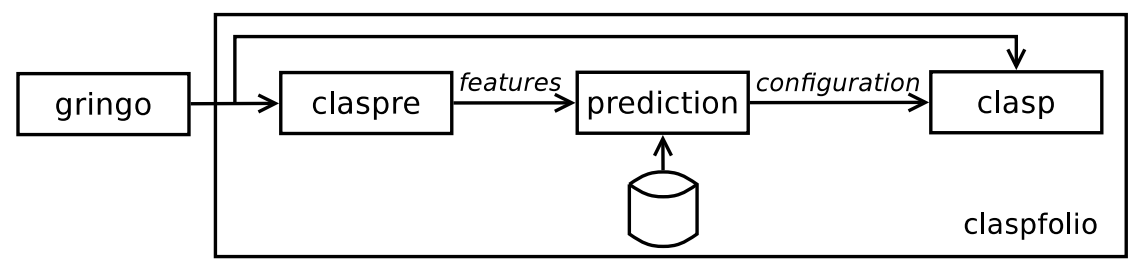

Fig. 1. Architecture of claspfolio

limited to some (manually) selected configurations belonging to a portfolio. Each configuration consists of certain clasp options, e.g., "--heuristic=VSIDS --local-restarts." To approximate the behavior of such a configuration, claspfolio applies a model-based approach predicting solving performance from particular features of the input.

As shown in Figure 1, ASP solving with claspfolio consists of four parts. First, the ASP grounder gringo [7] instantiates a logic program. Then, a light-weight version of clasp, called claspre, is used to extract features and possibly even solve a (too simple) instance. If the instance was not solved by claspre, the extracted features are mapped to a score for each configuration in the portfolio. Finally, clasp is run for solving, using the configuration with the highest score. Note that claspre and clasp use distinct copies of the input (see Figure 1) because preprocessing done by claspre may interfere with clasp options of configurations in the portfolio.

The features determined by claspre can be distinguished into data- and searchoriented ones. The former include 50 properties, such as number of constraints, number or variables, etc. Beyond that, claspre performs a limited amount of search to also collect information about solving characteristics. In this way, additional 90 search-related features are extracted, such as average backjump length, length of learned clauses, etc.

Given the features of an instance, claspfolio scores each configuration in the portfolio. To this end, it makes use of models generated by means of machine learning during a training phase. In the case of claspfolio, we applied Support Vector Regression, as implemented by the libSVM package [8]. Upon training, the score $s_{k}(i)$ of the $k$-th configuration on the $i$-th training instance is simply the runtime $t_{k}(i)$ in relation to the minimum runtime of all configurations in the portfolio: $s_{k}(i)=\frac{\min _{j}\left(t_{j}(i)\right)}{t_{k}(i)}$.

A model, i.e., a function mapping instance features to scores, is then generated from the feature-score pairs available for the training set. In production mode, only the features (collected by claspre), but not the configurations' scores, are available. Hence, the models are queried to predict the scores of all configurations in the portfolio, among which the one with the highest predicted score is selected for setting up clasp.

The portfolio used by claspfolio (version 0.8.0) contains 12 clasp configurations, included because of their complementary performances on the training set. The options of these configurations mainly configure the preprocessing, the decision heuristic, and the restart policy of clasp in different ways. This provides us with a collection of solving strategies that have turned out to be useful on a range of existing benchmarks. In fact, the hope is that some configuration is (a) well-suited for a user's application and (b) automatically selected by claspfolio in view of similarities to the training set. 


\section{Experiments}

We conducted experiments on benchmark classes of the 2009 ASP competition [9]. ${ }^{1}$ All experiments were run on an Intel Xeon E5520 machine, equipped with $2.26 \mathrm{GHz}$ processors and 48 GB RAM, under Linux. The considered systems are clasp (1.3.4) and claspfolio (0.8.0; based on clasp 1.3.4). Runtimes in seconds, per class and in total, are shown in Table 1. The first two columns give benchmark classes along with their numbers of instances (\#). The subsequent columns denote particular variants of the considered systems: clasp default (clasp), clasp manually tuned ${ }^{2}\left(\right.$ clasp $\left.^{m}\right)$, claspfolio running a random configuration (claspfolio ${ }^{r}$ ), claspfolio running the best configuration ${ }^{3}$ $\left(\right.$ claspfolio $\left.^{b}\right)$, claspfolio default (claspfolio) as available at [7], and claspfolio obtained by cross validation $\left(\right.$ claspfolio $\left.^{v}\right)$. The runtime per benchmark instance was limited to 1,200 seconds, and timeouts are taken as 1,200 seconds within accumulated results. The third last and the last column $(\times)$ in Table 1 provide the speedup of claspfolio and claspfolio $^{v}$, respectively, over clasp, i.e., the runtime of clasp divided by the one of claspfolio or claspfolio $^{v}$, per benchmark class (and in total in the last row).

The role of claspfolio $^{v}$ is to evaluate claspfolio on unseen instances. We do so by using 10-fold cross validation where the set of all available instances is randomly divided into a training set and a test set, consisting of 90 and 10 percent of the inspected instances, respectively. The regression models generated on the training set are then evaluated on the (unseen) test set. By repeating this procedure ten times, every instance is once solved based on models not trained on the instance.

Comparing clasp with clasp $^{m}$ in Table 1, manual tuning turns out to be mostly successful, and it decreases total runtime roughly by a factor of 3 . On two classes, Labyrinth and WireRouting, manual tuning was however counterproductive. This can be explained by the 2009 ASP competition mode, revealing only a few of the available instances per benchmark class during a setup phase, so that the manually tuned parameters may fail on unseen instances. In fact, claspfolio, trained on a collection of 3096 instances from the Asparagus benchmark repository ${ }^{4}$ and the 2009 ASP competition, turns out to be even more successful in total than clasp $^{m}$. In particular, it performs better on Labyrinth and WireRouting, where clasp $^{m}$ failed to improve over clasp. Of course, there are also benchmark classes on which manual tuning beats claspfolio (most apparently, WeightDomSet), but the fact that claspfolio exhibits a total speedup of 3.3 over clasp clearly shows the potential of automatic parameter selection. Notably, the total runtime of claspfolio exceeds the best possible one, claspfolio $^{b}$, only by a factor of 1.45, while the expected runtime of a random configuration, claspfolio ${ }^{r}$, is in total more than a factor of 4 greater than the one of claspfolio.

\footnotetext{
${ }^{1}$ Some too easy/unbalanced classes or instances, respectively, of the competition are omitted. On the other hand, we also ran additional instances for some classes. All instances used in our experiments are available at http://www.cs.uni-potsdam.de/claspfolio.

${ }^{2}$ The respective parameter settings per benchmark class are reported at http://dtai.cs . kuleuven.be/events/ASP-competition/Teams/Potassco.shtml.

${ }^{3}$ Note that results of claspfolio $^{r}$ and claspfolio $^{b}$ are calculated a posteriori per benchmark instance, using the average or smallest, respectively, runtime of all clasp variants in the portfolio.

${ }^{4}$ Available at http: // asparagus.cs.uni-potsdam.de.
} 


\begin{tabular}{l|r||r|r||r|r||r|r||r|r|}
\hline Benchmark Class & $\#$ & clasp & clasp $^{m}$ & claspfolio $^{r}$ & claspfolio $^{b}$ & claspfolio & $\times$ & claspfolio $^{v}$ & $\times$ \\
\hline \hline 15Puzzle & 37 & 510 & 281 & 438 & 111 & 208 & 2.4 & 254 & 2.0 \\
BlockedNQueens & 65 & 412 & 374 & 765 & 139 & 264 & 1.5 & 410 & 1.0 \\
ConnectDomSet & 21 & 1,428 & 54 & 1,236 & 30 & 53 & 26.9 & 649 & 2.2 \\
GraphColouring & 23 & 17,404 & 5,844 & 15,304 & 5,746 & 5,867 & 2.9 & 5,867 & 2.9 \\
GraphPartitioning & 13 & 135 & 66 & 791 & 57 & 69 & 1.9 & 97 & 1.4 \\
Hanoi & 29 & 458 & 130 & 499 & 35 & 175 & 2.6 & 233 & 2.0 \\
Labyrinth & 29 & 1,249 & 1,728 & 3.949 & 112 & 785 & 1.5 & 2,537 & 0.5 \\
MazeGeneration & 28 & 3,652 & 569 & 4,086 & 558 & 581 & 6.2 & 567 & 6.4 \\
SchurNumbers & 29 & 726 & 726 & 1,193 & 41 & 399 & 1.8 & 957 & 0.7 \\
Sokoban & 29 & 18 & 19 & 34 & 12 & 57 & 0.3 & 54 & 0.3 \\
Solitaire & 22 & 2,494 & 631 & 3,569 & 73 & 317 & 7.8 & 1,610 & 1.5 \\
WeightDomSet & 29 & 3,572 & 248 & 10,091 & 5 & 1,147 & 3.1 & 5,441 & 0.6 \\
WireRouting & 23 & 1,223 & 2,103 & 1,409 & 43 & 144 & 8.4 & 289 & 4.2 \\
\hline
\end{tabular}
Total Table 1. Runtimes in seconds and speedups on benchmark classes of the 2009 ASP competition

Comparing claspfolio, trained on all available instances, with claspfolio $^{v}$, where training and test sets are disjoint, we see that applying claspfolio $^{(v)}$ to unseen instances yields lower prediction quality. If the training set represents the dependencies between features and runtime rather loosely, the regression models hardly generalize to unseen instances, which obstructs a good parameter selection. But even in this case, claspfolio $^{v}$ is almost twice as fast as clasp, which shows that the trained models are still helpful.

In Table 2, we compare claspfolio with paramils, an automatic configuration tool based on iterated local search (FocusedILS) through the configuration space. Given that paramils uses a model-free approach, it can only generalize between homogeneous problem classes regarding the best configuration. In contrast, claspfolio is utterly applicable to heterogeneous classes in view of its regression models. To reflect this discrepancy, the column paramils ${ }^{c}$ shows the runtimes of the best configurations of clasp determined by paramils independently for each problem class, while the best configuration found over all problem classes is displayed in column paramils ${ }^{a}$. In both cases, we ran four (randomized) copies of paramils for 24 hours with a timeout of $600 \mathrm{sec}-$ onds per run on an instance, as suggested in [6], and then selected the best configuration found. Also note that, in view of only 377 instances evaluated overall, we did not split instances into a training and a test set, i.e., paramils was used to automatically analyze clasp configurations rather than predicting their performances.

As it could be expected, the configurations found by paramils $^{c}$ are much faster than the global one of paramils $^{a}$. On some problem classes, e.g., WeightDomSet, paramils ${ }^{c}$ found configurations that rendered the classes almost trivial to solve. On such classes, the configurations of paramils ${ }^{c}$ also yield much better performances than the ones of claspfolio and clasp $^{m}$. However, on problem classes including very hard instances, like GraphColouring and Solitaire, the configurations determined by paramils were less successful. This can be explained by long runs on instances, so that fewer configurations could be explored by local search within the allotted 24 hours. 


\begin{tabular}{|l|r||r|r||r|r||r|r|}
\hline Benchmark Class & $\#$ & paramil $^{c}$ & paramils $^{a}$ & claspfolio $_{\text {claspfolio }}$ & clasp $^{\text {clasp }}$ \\
\hline \hline 15Puzzle & 37 & 104 & 322 & 208 & 254 & 510 & 281 \\
BlockedNQueens & 65 & 212 & 352 & 264 & 410 & 412 & 374 \\
ConnectDomSet & 21 & 28 & 686 & 53 & 649 & 1,428 & 54 \\
GraphColouring & 23 & 7,596 & 10,865 & 5,867 & 5,867 & 17,404 & 5,844 \\
GraphPartitioning & 13 & 39 & 86 & 69 & 97 & 135 & 66 \\
Hanoi & 29 & 35 & 147 & 175 & 233 & 458 & 130 \\
Labyrinth & 29 & 462 & 3,080 & 785 & 2,537 & 1,249 & 1,728 \\
MazeGeneration & 28 & 700 & 2,610 & 581 & 567 & 3,652 & 569 \\
SchurNumbers & 29 & 278 & 871 & 399 & 957 & 726 & 726 \\
Sokoban & 29 & 11 & 18 & 57 & 54 & 18 & 19 \\
Solitaire & 22 & 2,374 & 4,357 & 317 & 1,610 & 2,494 & 631 \\
WeightDomSet & 29 & 8 & 2,649 & 1,147 & 5,441 & 3,572 & 248 \\
WireRouting & 23 & 87 & 535 & 144 & 289 & 1,223 & 2,103 \\
\hline Total & 377 & 11,934 & 26,578 & 10,066 & 18,965 & 33,281 & 12,773 \\
\hline
\end{tabular}

Table 2. Comparison with paramils on benchmark classes of the 2009 ASP competition

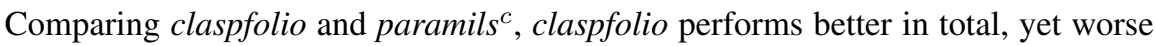
on ten of the thirteen classes. One reason is that claspfolio is based on a small set of configurations, whereas paramils considers a much larger configuration space (about $10^{12}$ configurations). In addition, paramils ${ }^{c}$ determined a suitable configuration individually for each class, while claspfolio applies the same configurations and models to all problem classes. In fact, we note that claspfolio $^{v}$ performs better than paramils ${ }^{a}$. From this, we conclude that the problem classes are heterogeneous, so that it is unlikely to find a single configuration well-suited for all classes. Thus, claspfolio appears to be a reasonable approach for configuring clasp for sets of heterogeneous instances.

\section{Discussion}

In this preliminary report, we described a simple yet effective way to counterbalance the sensitivity of ASP solvers to parameter configuration. As a result, ASP solving regains a substantial degree of declarativity insofar as users may concentrate on problem posing rather than parameter tuning. The resulting portfolio-based solver claspfolio largely improves on the default configuration of the underlying ASP solver clasp. Moreover, our approach outperforms a manual one conducted by experts.

Although our approach is inspired by satzilla, claspfolio differs in several ways. Apart from the different areas of application, SAT vs. ASP, satzilla's learning and selection engine relies on Ridge Regression, while ours uses Support Vector Regression. Interestingly, satzilla incorporates a SAT/UNSAT likelihood prediction further boosting its performance. Our first experiments in this direction did not have a similar effect, and it remains future work to investigate the reasons for this.

Our experiments emphasize that search for an optimal configuration, e.g., via paramils using local search, on one (homogeneous) problem class is more effective than claspfolio. But the search time of paramils for each problem class makes claspfolio more efficient on a set of (heterogeneous) problem classes. In fact, predicting a good configuration with claspfolio is almost instantaneous, once the regression models 
are trained. A recent approach to learn domain-specific decision heuristics [10] requires modifying a solver in order to learn and apply the heuristics.

It is interesting future work to investigate automatic portfolio generation. New configurations, to add to a portfolio, could be found with paramils. First attempts are done with hydra [11]. Further related work includes [12-16], whose discussion is however beyond the scope of this paper. Another goal of future work includes the investigation and selection of the extracted features to predict more precisely the runtime. Usually, feature selection decreases the prediction error of machine learning algorithms. In view of this, the potential of claspfolio is not yet fully harnessed in its current version.

Acknowledgments. This work was partly funded by DFG grant SCHA 550/8-2. We are grateful to Holger H. Hoos and Frank Hutter for fruitful discussions on the subject of this paper.

\section{References}

1. Baral, C.: Knowledge Representation, Reasoning and Declarative Problem Solving. Cambridge University (2003)

2. Gebser, M., Kaufmann, B., Neumann, A., Schaub, T.: Conflict-driven answer set solving. In IJCAI'07, AAAI Press (2007) 386-392

3. Pipatsrisawat, K., Darwiche, A.: On the power of clause-learning SAT solvers with restarts. In CP'09, Springer (2009) 654-668

4. Xu, L., Hutter, F., Hoos, H., Leyton-Brown, K.: SATzilla: Portfolio-based algorithm selection for SAT. JAIR 32 (2008) 565-606

5. Basak, D., Pal, S., Patranabis, D.: Support vector regression. NIP 11(10) (2007) 203-224

6. Hutter, F., Hoos, H., Leyton-Brown, K., Stützle, T.: ParamILS: An automatic algorithm configuration framework. JAIR 36 (2009) 267-306

7. http://potassco.sourceforge. net

8. http://www.csie.ntu.edu.tw/ cjlin/libsvm

9. Denecker, M., Vennekens, J., Bond, S., Gebser, M., Truszczyński, M.: The second answer set programming competition. In LPNMR'09, Springer (2009) 637-654

10. Balduccini, M.: Learning domain-specific heuristics for answer set solvers. In ICLP'10 Tech. Comm. (2010) 14-23

11. $\mathrm{Xu}, \mathrm{L} ., \mathrm{Hoos}, \mathrm{H}$., Leyton-Brown, K.: Hydra: Automatically configuring algorithms for portfolio-based selection. In AAAI'10, AAAI Press (2010) 210-216

12. Gagliolo, M., Schmidhuber, J.: Learning dynamic algorithm portfolios. AMAI 47(3-4) (2006) 295-328

13. Samulowitz, H., Memisevic, R.: Learning to solve QBF. In AAAI'07, AAAI Press (2007) 255-260

14. O’Mahony, E., Hebrard, E., Holland, A., Nugent, C., O'Sullivan, B.: Using case-based reasoning in an algorithm portfolio for constraint solving. In AICS'08 (2008)

15. Pulina, L., Tacchella, A.: A self-adaptive multi-engine solver for quantified Boolean formulas. Constraints 14(1) (2009) 80-116

16. Arbelaez, A., Hamadi, Y., Sebag, M.: Continuous search in constraint programming. In ICTAI' 10, IEEE Press (2010) 53-60 